\title{
Non-Abelian dual Meissner effect and confinement/deconfinement phase transition in SU(3) Yang-Mills theory
}

\author{
Akihiro Shibata* \\ Computing Research Center, High Energy Accelerator Research Organization (KEK) \& \\ Graduate University for Advanced Studies (Sokendai), Tsukuba 305-0801, Japan \\ E-mail: akihiro.shibata@kek.jp

\section{Kei-Ichi Kondo} \\ Department of Physics, Graduate School of Science, Chiba University, Chiba 263-8522, Japan \\ E-mail: kondok@faculty.chiba-u.jp
}

\section{Seikou Kato}

Fukui National College of Technology, Sabae, Fukui 916-8507, Japan

E-mail: skato@fukui-nct.ac.jp

\section{Toru Shinohara}

Department of Physics, Graduate School of Science, Chiba University, Chiba 263-8522, Japan

E-mail: sinohara@graduate.chiba-u.jp

\begin{abstract}
The dual superconductivity is a promising mechanism for quark confinement. We proposed the nonAbelian dual superconductivity picture for SU(3) Yang-Mills theory, and demonstrated the restricted field dominance (called conventionally "Abelian" dominance), and non-Abelian magnetic monopole dominance in the string tension. In the last conference, we have demonstrated by measuring the chromoelectric flux that the non-Abelian dual Meissner effect exists and determined that the dual superconductivity for $\mathrm{SU}(3)$ case is of type I, which is in sharp contrast to the $\mathrm{SU}(2)$ case: the border of type I and type II. In this talk, we focus on the confinement/deconfinemen phase transition and the non-Abelian dual superconductivity at finite temperature: We measure the chromoelectric flux between a pair of static quark and antiquark at finite temperature, and investigate its relevance to the phase transition and the non-Abelian dual Meissner effect.
\end{abstract}

31st International Symposium on Lattice Field Theory LATTICE 2013

July 29 - August 3, 2013

Mainz, Germany 


\section{Introduction}

Quark confinement follows from the area law of the Wilson loop average. The dual superconductivity is the promising mechanism for the quark confinement [1]. Based on the Abelian projection, there have been many numerical analyses to show evidences such as Abelian dominance [2], Abelian magnetic monopole dominance [3], and center vortex dominance [4] in the string tension. However, these results are obtained only in special gauges such as the maximal Abelian (MA) gauge and the Laplacian Abelian gauge, and the Abelian projection itself breaks the gauge symmetry as well as color symmetry (global symmetry).

We have presented the lattice version of a new formulation of $S U(N)$ Yang-Mills (YM) theory[5], that gives the decomposition of the gauge link variable $U_{x, \mu}=X_{x, \mu} V_{x, \mu}$, which is suited for extracting the dominant mode, $V_{x, \mu}$, for quark confinement in the gauge independent way. In the case of the $S U(2) \mathrm{YM}$ theory, the decomposition of the gauge link variable is given by a compact representation of Cho-DuanGe-Faddeev-Niemi (CDGFN) decomposition [6] on a lattice [7][8][9]. For the $S U(N)$ YM theory, the new formula for the decomposition of the gauge link variable is constructed as an extension of the $S U(2)$ case.

To the Wilson loop in the fundamental representation, we have apply the minimal option. The minimal option is obtained for the stability group of $\tilde{H}=U(2) \cong S U(2) \times U(1)$, which is suitable for the Wilson loop in the fundamental representation. This fact is derived from the non-Abelian Stokes' theorem [21]. Then, we have demonstrated the gauge independent (invariant) restricted $V$-field dominance, (or conventionally called Abelian dominance): the decomposed $V$-field (restricted field) reproduced the string tension of original YM field $\left(\sigma_{V} / \sigma_{\text {full }}=93 \pm 16 \%\right)$, and the gauge independent non-Abelian magnetic monopole dominance: the string tension was reproduced by only the (non-Abelian) magnetic monopole part extracted from the restricted field, $\left(\sigma_{m o n} / \sigma_{V}=94 \pm 9 \%\right)$ [13][11][14][15][20].

To establish the dual superconductivity picture, we must also show the magnetic monopoles play the dominant role in quark confinement. The dual Meissner effect in Yang-Mills theory must be examined by measuring the distribution of chromoelectric field strength or chromo flux as well as magnetic monopole currents created by a static quark-antiquark pair [16]. In $S U(2)$ case, the extracted field corresponding to the stability group $\tilde{H}=U(1)$ shows the dual Meissner effect, which is a gauge invariant version of the Abelian projection in MA gauge. In the SU(3) case, there are many works on chromo flux for the YangMills field by using Wilson line/loop operator, e.g., [23][24][25]. However, there is no direct measurement of the dual Meissner effect in the gauge independent (invariant) way, except for several studies based on the Abelian projection, e.g., [27]. At the last conference, we have demonstrated the non-Abelian dual Meissner effect[16]. By applying our new formulation to the $S U$ (3) YM theory, we have given the evidence of the non-Abelian dual Meissner effect claimed by us, and found the chromoelectric flux tube by measuring the chromo flux created by a static quark-antiquark pair. We have determined that the type of vacuum for $\mathrm{SU}(3)$ case is of type I, which is in sharp contrast to the $S U(2)$ case: the border of type I and type II.[18].

In this talk, we focus on the confinement/deconfinement phase transition and the non-Abelian dual superconductivity at finite temperature: We measure a Polyakov loop average and correlation functions of the Polyakov loops which are defined for both the original YM field and extracted $V$-field to examine the $V$-field dominance in the Polyakov loop at finite temperature. Then, we measure the chromoelectric flux between a pair of static quark and antiquark at finite temperature, and investigate its relevance to the phase transition and the non-Abelian dual Meissner effect. 


\section{Method}

We introduce a new formulation of the lattice YM theory in the minimal option, which extracts the dominant mode of the quark confinement for $S U(3)$ YM theory[20, 15], since we consider the quark confinement in the fundamental representation. Let $U_{x, \mu}=X_{x, \mu} V_{x, \mu}$ be the decomposition of YM link variable $U_{x, \mu}$, where $V_{x, \mu}$ could be the dominant mode for quark confinement, and $X_{x, \mu}$ the remainder part. The YM field and the decomposed new variables are transformed by full $S U(3)$ gauge transformation $\Omega_{x}$ such that $V_{x, \mu}$ is transformed as the gauge link variable and $X_{x, \mu}$ as the site variable:

$$
\begin{aligned}
U_{x, \mu} \longrightarrow U_{x, v}^{\prime} & =\Omega_{x} U_{x, \mu} \Omega_{x+\mu}^{\dagger}, \\
V_{x, \mu} \longrightarrow V_{x, v}^{\prime} & =\Omega_{x} V_{x, \mu} \Omega_{x+\mu}^{\dagger}, X_{x, \mu} \longrightarrow X_{x, v}^{\prime}=\Omega_{x} X_{x, \mu} \Omega_{x}^{\dagger} .
\end{aligned}
$$

The decomposition is given by solving the defining equation:

$$
\begin{aligned}
& D_{\mu}^{\varepsilon}[V] \mathbf{h}_{x}:=\frac{1}{\varepsilon}\left[V_{x, \mu} \mathbf{h}_{x+\mu}-\mathbf{h}_{x} V_{x, \mu}\right]=0, \\
& g_{x}:=e^{i 2 \pi q / 3} \exp \left(-i a_{x}^{0} \mathbf{h}_{x}-i \sum_{j=1}^{3} a_{x}^{(j)} \mathbf{u}_{x}^{(j)}\right)=1,
\end{aligned}
$$

where $\mathbf{h}_{x}$ is an introduced color field $\mathbf{h}_{x}=\xi\left(\lambda^{8} / 2\right) \xi^{\dagger} \in[S U(3) / U(2)]$ with $\lambda^{8}$ being the Gell-Mann matrix and $\xi$ an $S U(3)$ group element. The variable $g_{x}$ is an undetermined parameter from Eq.(2.2a), $\mathbf{u}_{x}^{(j)}$,s are $s u(2)$-Lie algebra values, and $q_{x}$ an integer value $0,1,2$. These defining equations can be solved exactly [19], and the solution is given by

$$
\begin{array}{ll}
X_{x, \mu}=\widehat{L}_{x, \mu}^{\dagger} \operatorname{det}\left(\widehat{L}_{x, \mu}\right)^{1 / 3} g_{x}^{-1}, & V_{x, \mu}=X_{x, \mu}^{\dagger} U_{x, \mu}=g_{x} \widehat{L}_{x, \mu} U_{x, \mu}, \\
\widehat{L}_{x, \mu}=\left(L_{x, \mu} L_{x, \mu}^{\dagger}\right)^{-1 / 2} L_{x, \mu}, & L_{x, \mu}=\frac{5}{3} \mathbf{1}+\frac{2}{\sqrt{3}}\left(\mathbf{h}_{x}+U_{x, \mu} \mathbf{h}_{x+\mu} U_{x, \mu}^{\dagger}\right)+8 \mathbf{h}_{x} U_{x, \mu} \mathbf{h}_{x+\mu} U_{x, \mu}^{\dagger} .
\end{array}
$$

Note that the above defining equations correspond to the continuum version: $D_{\mu}[\mathscr{V}] \mathbf{h}(x)=0$ and $\operatorname{tr}\left(\mathbf{h}(x) \mathscr{X}_{\mu}(x)\right)$ $=0$, respectively. In the naive continuum limit, we have the corresponding decomposition $\mathbf{A}_{\mu}(x)=$ $\mathbf{V}_{\mu}(x)+\mathbf{X}_{\mu}(x)$ in the continuum theory[19] as

$$
\begin{aligned}
& \mathbf{V}_{\mu}(x)=\mathbf{A}_{\mu}(x)-\frac{4}{3}\left[\mathbf{h}(x),\left[\mathbf{h}(x), \mathbf{A}_{\mu}(x)\right]\right]-i g^{-1} \frac{4}{3}\left[\partial_{\mu} \mathbf{h}(x), \mathbf{h}(x)\right], \\
& \mathbf{X}_{\mu}(x)=\frac{4}{3}\left[\mathbf{h}(x),\left[\mathbf{h}(x), \mathbf{A}_{\mu}(x)\right]\right]+i g^{-1} \frac{4}{3}\left[\partial_{\mu} \mathbf{h}(x), \mathbf{h}(x)\right] .
\end{aligned}
$$

The decomposition is uniquely obtained as the solution (2.3) of Eqs.(2.2), if color fields $\left\{\mathbf{h}_{x}\right\}$ are obtained. To determine the configuration of color fields, we use the reduction condition to formulate the new theory written by new variables $\left(X_{x, \mu}, V_{x, \mu}\right)$ which is equipollent to the original YM theory. Here, we use the reduction functional:

$$
F_{\mathrm{red}}\left[\mathbf{h}_{x}\right]=\sum_{x, \mu} \operatorname{tr}\left\{\left(D_{\mu}^{\varepsilon}\left[U_{x, \mu}\right] \mathbf{h}_{x}\right)^{\dagger}\left(D_{\mu}^{\varepsilon}\left[U_{x, \mu}\right] \mathbf{h}_{x}\right)\right\}
$$

and then color fields $\left\{\mathbf{h}_{x}\right\}$ are obtained by minimizing the functional (2.5).

\section{Result}

We generate YM gauge configurations $\left\{U_{x, \mu}\right\}$ at finite temperature using the standard Wilson action on lattices $L^{3} \times N_{T}: L=24, N_{T}=6,8,10,14,24$ with $\beta=6.0, L=24, N_{T}=4,6,8,10,12,14,24$ with 

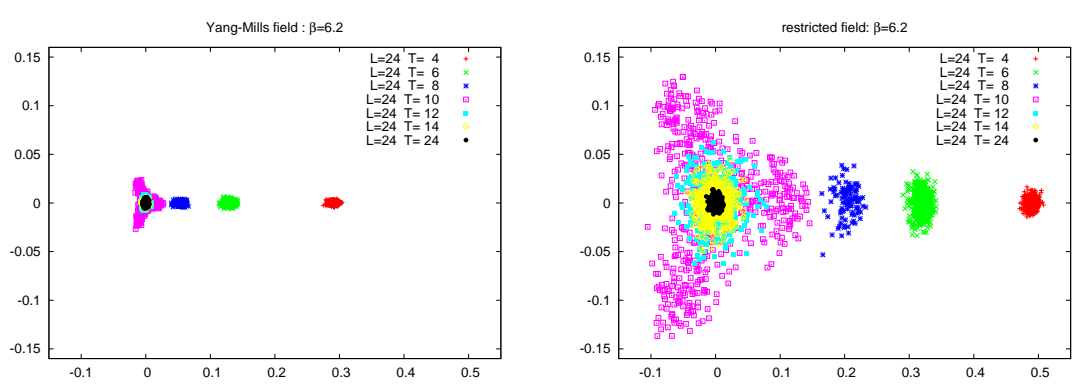

Figure 1: The distribution of the space-averaged Polyakov loop for each ocnfiguration, Eq.(3.1) (Left) For the YM field. (Right) For the restricted field.
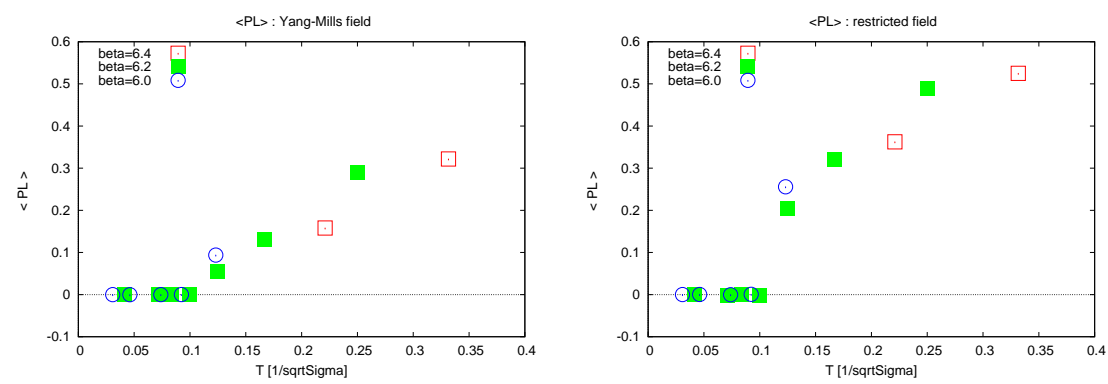

Figure 2: The Polyakov loop average: (Left) For the YM field $\left\langle P_{U}\right\rangle$. (Right) For the restricted field $\left\langle P_{V}\right\rangle$.

$\beta=6.2$ and $L=24, N_{T}=4,6$ with $\beta=6.4$. The gauge link decomposition $U_{x, \mu}=X_{x, \mu} V_{x, \mu}$ is obtained by the formula (2.3) given in the previous section, after the color field configuration $\left\{\mathbf{h}_{x}\right\}$ is obtained by solving the reduction condition of minimizing the functional eq(2.5) for each gauge configuration. In the measurement of the Polyakov loop and Wilson loop, we apply the APE smearing technique to reduce noises [29].

Figure 1 show the distribution of space-averaged Polyakov loops for each configurations:

$$
P_{U}:=L^{-3} \sum \operatorname{tr}\left(\prod_{t=1}^{N_{T}} U_{(\vec{x}, t), 4}\right), \quad P_{V}:=L^{-3} \sum \operatorname{tr}\left(\prod_{t=1}^{N_{T}} V_{(\vec{x}, t), 4}\right) .
$$

The left panel shows the distribution of the YM field and the right panel that of the restricted field ( $V$-field). Then, we obtain the Polyakov loop average for configurations, which is the conventional order parameter for confinement and deconfinement phase transition in $S U$ (3) YM theory. Figure 2 shows the Polyakov loop average for the YM field $\left\langle P_{U}\right\rangle$ (left panel) and restricted field $\left\langle P_{V}\right\rangle$ (right panel). Each panel shows the same critical temperature of confinement/deconfinement phase transition. Then, we investigate two-point correlation function of the Polyakov loop:

$$
D_{U}(x-y):=\left\langle P_{U}(x)^{*} P_{U}(y)\right\rangle-\left\langle\left|P_{U}\right|^{2}\right\rangle, \quad D_{V}(x-y):=\left\langle P_{V}(x)^{*} P_{V}(y)\right\rangle-\left\langle\left|P_{V}\right|^{2}\right\rangle,
$$

Figure 3 shows the comparison of the $D_{U}(x-y)$ and $D_{V}(x-y)$ each temperature. Every panel shows that the YM-field and restricted field ( $V$-field) have the same profile, i.e., we can extract the dominant mode for the quark confinement by the $V$-field.

Next, we investigate the non-Abelian dual Meissner effect at finite temperature. To investigate the chromo flux, we use the gauge invariant correlation function which is used at zero temperature. The chromo flux created by a quark-antiquark pair is measured by using a gauge-invariant connected correlator 

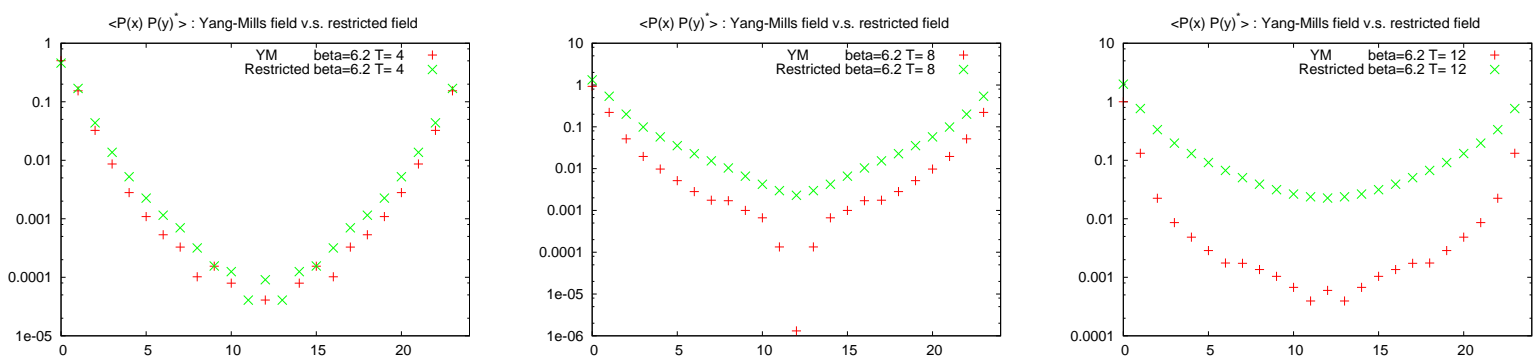

Figure 3: Comparison of the correlation function of the Polyakov loop for the YM field and the restricted field. (Left) At high temperature. (Middle) Near critical temperature. (Right) At low temperature.
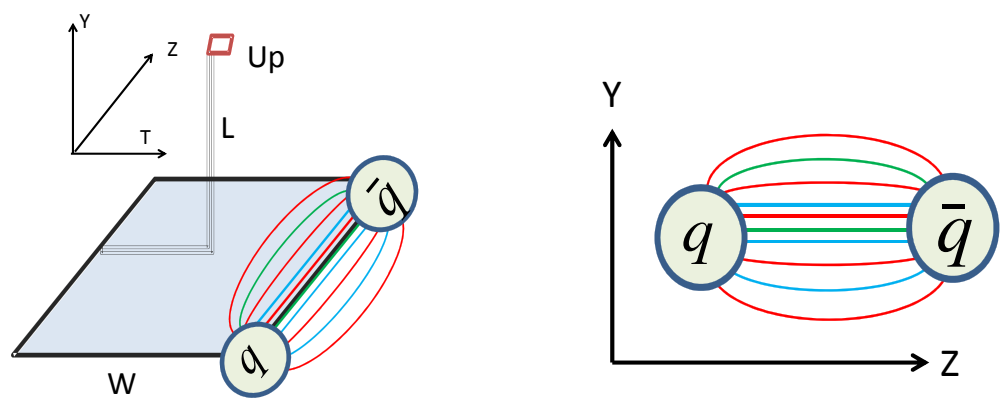

Figure 4: (Left) The connected correlator $\left(U_{p} L W L^{\dagger}\right)$ between a plaquette $U_{p}$ and the Wilson loop $W$. (Right) Measurement of the chromo-flux in the Y-Z plane.

of the Wilson loop [26]:

$$
\rho_{W}:=\frac{\left\langle\operatorname{tr}\left(U_{p} L^{\dagger} W L\right)\right\rangle}{\langle\operatorname{tr}(W)\rangle}-\frac{1}{3} \frac{\left\langle\operatorname{tr}\left(U_{p}\right) \operatorname{tr}(W)\right\rangle}{\langle\operatorname{tr}(W)\rangle},
$$

where $W$ represents a quark-antiquark pair settled by the Wilson loop in Z-T plane, $U_{p}$ a plaquette variable as the probe operator for measuring the field strength, and $L$ the Wilson line connecting the source $W$ and the probe $U_{p}$. (see the left panel of Figure 4). The symbol $\langle\mathscr{O}\rangle$ denotes the average of the operator $\mathscr{O}$ over the space and the ensemble of the configurations. Note that this is sensitive to the field strength rather than the disconnected one. Indeed, in the naive continuum limit, the connected correlator $\rho_{W}$ is given by $\rho_{W} \stackrel{\varepsilon \rightarrow 0}{\simeq} g \varepsilon^{2}\left\langle\mathscr{F}_{\mu v}\right\rangle_{q \bar{q}}:=\frac{\left\langle\operatorname{tr}\left(g \varepsilon^{2} \mathscr{F}_{\mu v} L^{\dagger} W L\right)\right\rangle}{\langle\operatorname{tr}(W)\rangle}+O\left(\varepsilon^{4}\right)$. Thus, the chromo field strength is given by $F_{\mu \nu}=\sqrt{\frac{\beta}{6}} \rho_{W}$. Note that at finite temperature we must use the operator with the same size in the temporal direction, and the quark and antiquark pair is replaced by a pair of the Polyakov loop with the opposite direction.

Figure 5 shows the measurement of the Z-component $E_{z}$ of chromoelectric flux at zero temperature. We observe the chromoelectric flux tube only in the direction connecting quark and antiquark pair, while the other components take vanishing values [18]. Figure 6] shows the measurement of chromoelectric and chromomagnetic flux at high temperature $T>T_{c}$ (for the lattice $N_{T}=6, \beta=6.2$ ). We measure the chromo-flux of quark-antiquark pair in the plane $z=1 / 3 R$ for a quark at $z=0$ and an antiquark at $z=R$ (Fig.4) by moving the probe, $U_{p}$ or $V_{p}$ along the y-direction. We can observe no more squeezing of the chromoelectric flux tube, but non-vanishing $E_{y}$ component in the chromoelectric flux. This shows the disappearance of the dual Meisner effect at high temperature. 

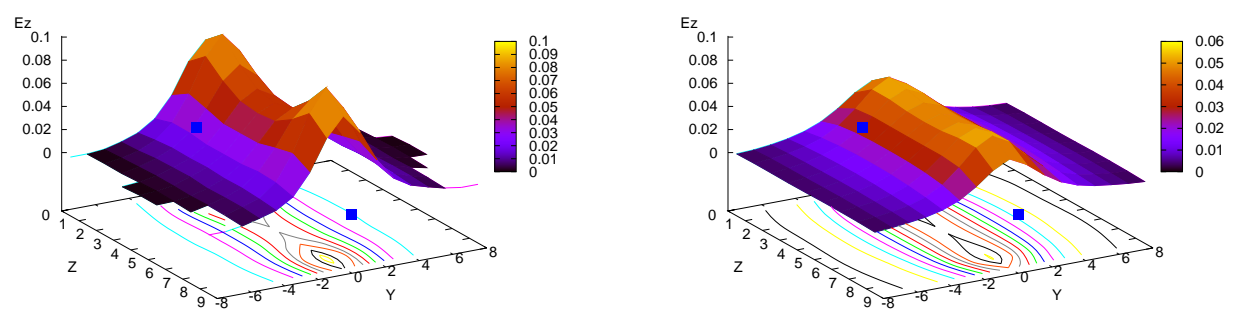

Figure 5: The distribution in $Y-Z$ plane of the chromoelectric field $E_{z}$ connecting a pair of quark and antiquark: (Left panel) chromoelectric field produced from the original YM field, (Right panel) chromoelectric field produced from the restricted field.
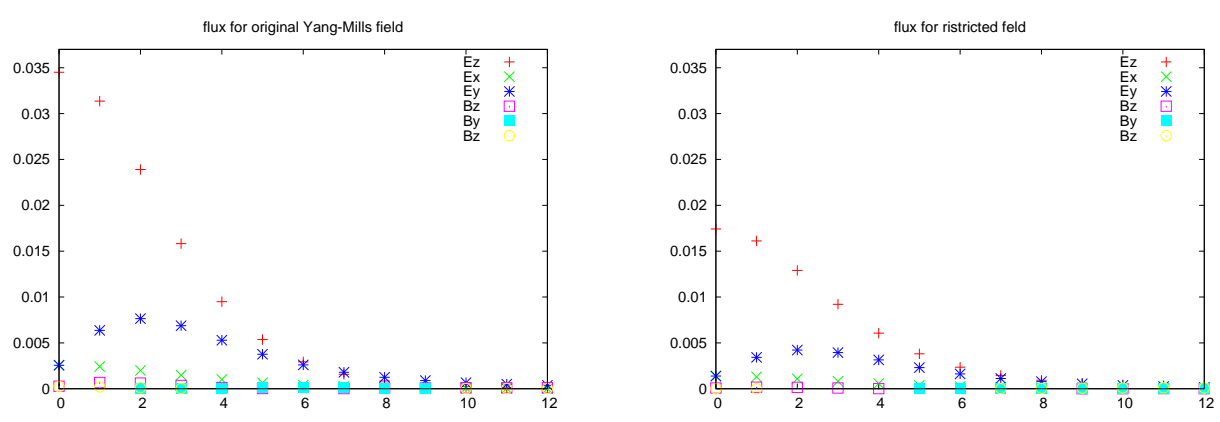

Figure 6: The chromo-flux created by the quark-antiquark pair in the plane $z=1 / 3 R$ for a quark at $z=0$ and an antiquark at $z=R(\mathrm{Fig}, 4)$ by moving the probe, $U_{p}$ or $V_{p}$ along the y-direction. (Left) For the YM-field. (Right) For the restricted field.

\section{Summary and outlook}

We have studied the dual superconductivity for $S U(3)$ YM theory by using our new formulation of YM theory on a lattice. We have extracted the restricted field ( $V$-field) from the YM field which plays a dominant role in confinement of quark (fermion in the fundamental representation) at finite temperature, i.e., the restricted field dominance in Polyakov loop. Then we have measured the chromoelectric and chromomagnetic flux for both the original YM field and the restricted field at low temperature. We have observed evidences of the dual Messier effect of $S U$ (3) YM theory, i.e., the chromoelectric flux tube and the associated non-Abelian magnetic monopoles created by quark and antiquark pair. At high temperature $\left(T>T_{c}\right)$ in the deconfinement phase, we have observed the disappearance of the dual Meissner effect by measuring the chromo flux. Note that, the Polyakov loop average cannot be the direct signal of the dual Meissner effect or magnetic monopole condensation. Therefore, it is important to find a order parameter which detect the dual Meissner effect directly, and to investigate whether or not the order parameter in view of the dual Meissner effect gives the same critical temperature as that of the Polyakov loop average.

\section{Acknowledgement}

This work is supported by Grant-in-Aid for Scientific Research (C) 24540252 from Japan Society for the Promotion Science (JSPS), and also in part by JSPS Grant-in-Aid for Scientific Research (S) 22224003. The numerical calculations are supported by the Large Scale Simulation Program No.12-13 (FY2012), No. 12/13-20 (FY2012/13) of High Energy Accelerator Research Organization (KEK). 


\section{References}

[1] Y. Nambu, Phys. Rev. D10, 4262(1974); G. 't Hooft, in: High Energy Physics, edited by A.; Zichichi (Editorice Compositori, Bologna, 1975).; S. Mandelstam, Phys. Report 23, 245(1976).; A.M. Polyakov, Nucl. Phys. B120, 429(1977).

[2] T. Suzuki and I. Yotsuyanagi, Phys. Rev. D42 4275 (1990)

[3] J.D.Stack, S.D. Neiman and R.Wensley, Phys. Rev. D50 3399 (1994); H. Shiba and T. Suzuki, Phys. Lett. B351 519 (1995)

[4] J. Greensite, Prog. Part. Nucl. Phys. 511 (2003)

[5] K.-I. Kondo, T. Murakami and T. Shinohara, Eur. Phys. J. C 42, 475 (2005); K.-I. Kondo, T. Murakami and T. Shinohara, Prog. Theor. Phys. 115, 201 (2006).; K.-I. Kondo, T. Shinohara and T. Murakami, Prog.Theor. Phys. 120, 1 (2008)

[6] Y.M. Cho, Phys. Rev. D 21, 1080 (1980). Phys. Rev. D 23, 2415 (1981); Y.S. Duan and M.L. Ge, Sinica Sci., 11, 1072(1979); L. Faddeev and A.J. Niemi, Phys. Rev. Lett. 82, 1624 (1999); S.V. Shabanov, Phys. Lett. B 458, 322 (1999). Phys. Lett. B 463, 263 (1999).

[7] S. Ito, S. Kato, K.-I. Kondo, A. Shibata, T. Shinohara, Phys.Lett. B645 67-74 (2007)

[8] A. Shibata, S. Kato, K.-I. Kondo, T. Murakami, T. Shinohara, S. Ito, Phys.Lett. B653 101-108 (2007)

[9] S. Kato, K.-I. Kondo, A. Shibata and T. Shinohara, PoS(LAT2009) 228.

[10] A. Shibata, S. Kato, K.-I. Kondo, T. Shinohara and S. Ito, POS(LATTICE2007) 331, arXiv:0710.3221 [hep-lat]

[11] A. Shibata, S. Kato, K.-I. Kondo, T. Shinohara and S. Ito, PoS(LATTICE 2008) 268, arXiv:0810.0956 [hep-lat]

[12] Shinya Gongyo, Takumi Iritani, Hideo Suganuma, Phys.Rev. D86 (2012) 094018

[13] K.-I. Kondo, A.Shibata, T. Shinohara, T. Murakami, S. Kato and S. Ito, Phys. Lett. B669, 107 (2008)

[14] A. Shibata, K.-I. Kondo, S.Kato, S. Ito, T. Shinohara, N. Fukui, PoS LAT2009 (2009) 232, arXiv:0911.4533 [hep-lat].

[15] A. Shibata, K.-I. Kondo, S. Kato and T. Shinohara, PoS(Lattice 2010)286

[16] A. Shibata, K.-I. Kondo, S. Kato and T. Shinohara, PoS LATTICE2012 (2012) 215

[17] A. Shibata, K.-I. Kondo, S. Kato and T. Shinohara, PoS ConfinementX (2012) 052, : arXiv:1302.6865 [hep-lat]

[18] A. Shibata, K.-I. Kondo, S. Kato and T. Shinohara, Phys.Rev. D87 (2013) 5, 054011, arXiv:1212.6512[hep-lat]

[19] A. Shibata, K.-I. Kondo and T. Shinohara, Phys.Lett.B691:91-98,2010, arXiv:0911.5294[hep-lat]

[20] K.-I. Kondo, A. Shibata, T. Shinohara, S. Kato, Phys.Rev. D83 (2011) 114016, arXiv:1007.2696 [hep-th]

[21] K.-I. Kondo, Phys.Rev.D77 085029 (2008)

[22] K.-I. Kondo and A. Shibata, CHIBA-EP-170, KEK-PREPRINT-2007-73, arXiv:0801.4203[hep-th]

[23] M. S. Cardaci, P. Cea, L. Cosmai, R. Falcone and A. Papa, Phys.Rev.D83:014502, 2011

[24] N. Cardoso, M. Cardoso, P. Bicudo, arXiv:1107.1355 [hep-lat] (also lattice2011)

[25] Cea, Cosmai and Papa, PRD86(054501) 2012

[26] A. Di Giacomo, M. Maggiore, and S. Olejnik, Phys. Lett. B236, 199 (1990); Nucl. Phys. B347, 441 (1990).

[27] Y. Matsubara, S. Ejiri and T. Suzuki, NPB Poc. suppl 34, 176 (1994)

[28] R.G.Edwards, U.M. Heller and T.R.Klassen, Phys. Rev. Lett. 80, 3448-3451 (1998)

[29] M. Albanese et al. (APE Collaboration), Phys. Lett. B 192,163 (1987). 